Narrowing in face and speech perception in infancy:

Developmental change in the relations between domains

\author{
${ }^{*}$ Naiqi G. Xiao ${ }^{1}$ \\ Mai Mukaida ${ }^{2}$ \\ Paul C. Quinn ${ }^{3}$ \\ Olivier Pascalis ${ }^{4}$ \\ Kang Lee ${ }^{1}$

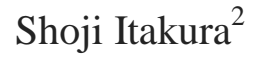

1. Dr. Eric Jackman Institute of Child Study, University of Toronto, Toronto, Canada

2. Department of Psychology, Graduate School of Letters, Kyoto University, Kyoto, Japan

3. Department of Psychological and Brain Sciences, University of Delaware, Newark, USA

4. LPNC - Université Grenoble Alpes, CNRS, Grenoble, France

\title{
Acknowledgements
}

This research was supported by grants from the Natural Science Engineering Research Council of Canada, National Institutes of Health (R01 HD046526), and Japan Society for the Promotion of Science Grants (25245067 and 25240020).

\footnotetext{
* Naiqi G. Xiao and Mai Mukaida made equal contributions to the current paper.
} 


\title{
Narrowing in face and speech perception in infancy: \\ Developmental change in the relations between domains
}

\begin{abstract}
Although prior research has established that perceptual narrowing reflects the influence of experience on the development of face and speech processing, it is unclear whether narrowing in the two domains is related. A within-subject design $(N=72)$ was used to investigate discrimination of own- and other-race faces and native and non-native speech sounds in 3-, 6-, 9-, and 12-month-olds. For face and speech discrimination, whereas 3-month-olds discriminated own-race faces and native speech sounds, and other-race faces and non-native speech sounds, older infants only discriminated own-race faces and native speech sounds. Narrowing in face and speech were not correlated at 6 months, negatively correlated at 9 months, and positively correlated at 12 months. The findings demonstrated developmental change in the relation between modalities during the first year. Implications for domain-general versus domain-specific accounts of perceptual development are discussed.
\end{abstract}

keywords: perceptual narrowing; face perception; speech perception; perceptual development; infancy 


\section{Narrowing in face and speech perception in infancy: \\ Developmental change in relations between domains}

Experience exerts strong influence on the development of perception in infancy. Such influence can be observed in an apparently regressive age-related change in infant discrimination of familiar and unfamiliar stimuli: Young infants initially can discriminate differences among familiar stimuli and also among unfamiliar stimuli. However, with increased age and related increase in experience with processing familiar stimuli, infants maintain the ability to discriminate differences among familiar stimuli, but begin to experience difficulty differentiating among unfamiliar stimuli (Kelly et al., 2007; Kuhl, Tsao, \& Liu, 2003; Kuhl, Williams, Lacerda, Stevens, \& Lindblom, 1992; Le Grand, Mondloch, Maurer, \& Brent, 2001; Macchi Cassia, Bulf, Quadrelli, \& Proietti, 2013; Pascalis, de Haan, \& Nelson, 2002; Pascalis et al., 2005; Pons, Lewkowicz, Soto-Faraco, \& Sebastián-Gallés, 2009; Werker \& Tees, 1984). This experience-related developmental pattern in infancy is referred to broadly as perceptual narrowing (for a review, see Maurer \& Werker, 2014).

Narrowing has been consistently observed in various domains, such as face, speech, music, and intermodal perception. The similarity in the narrowing among different perceptual domains suggests that narrowing may be driven by domain-general mechanisms, that are not specific to a particular perceptual domain (Pascalis et al., 2014; Scott, Pascalis, \& Nelson, 2007). However, because nearly all prior investigations have studied narrowing only in a single domain, there is little work that bears on the domain-general account. It is therefore entirely unknown whether narrowing in one domain is related to that in another domain, as suggested by the domain-general hypothesis. To bridge this significant gap in the literature, the current study 
investigated the relations between narrowing in face and speech perception in infants at 3, 6, 9, and 12 months of age with a within-subject design.

For the domain of faces, face race is perhaps the most well-studied category to illustrate the influence of experience on the development face perception (Kelly et al., 2007, 2009, see Sugden \& Marquis, 2017 for a recent review). In the first year of life, infants are mostly exposed to faces from their own race, while having limited access to other-race faces (Liu et al., 2015; Rennels \& Davis, 2008; Sugden, Mohamed-Ali, \& Moulson, 2014). As a result of this asymmetrical face-race experience, perceptual narrowing occurs in the processing of own- and other-race faces (Kelly et al., 2007, 2009; Liu et al., 2011; Quinn, Lee, Pascalis, \& Tanaka, 2016; Tham, Bremner, \& Hay, 2015; Wheeler et al., 2011; but see Chien, Wang, \& Huang, 2016). For example, Kelly and colleagues (2007) found that 3-month-old Caucasian infants could discriminate among own-race faces and also among other-race faces. By contrast, 9-month-old Caucasian infants were only able to discriminate among own-race Caucasian faces, but not within other-race face classes, such as Asian or African faces. This developmental pattern of responding to own- versus other-race faces was later replicated with Asian infants (Kelly et al., 2009). Moreover, consistent with the experiential account of narrowing, experimentally exposing infants to other-race faces was found to prevent and even reverse narrowing for other-race faces (Anzures et al., 2012; Heron-Delaney et al., 2011). Similar perceptual narrowing patterns have been found in processing other aspects of facial information such as species and age (Kobayashi, Macchi Cassia, Kanazawa, Yamaguchi, \& Kakigi, 2016; Macchi Cassia et al., 2013; Pascalis et al., 2002; Scott \& Monesson, 2009). Thus, it is now well established that narrowing in face perception is a common phenomenon in infancy that reflects the experience-based tuning of the 
perceptual system to optimally process faces of the most frequently encountered categories in the visual environment of infants.

Similar to their experience with faces, infants also have asymmetrical experience with native versus non-native speech. They typically have far greater exposure to native speech than non-native speech. This asymmetrical experience with speech is known to shape the early development of speech perception, also through a narrowing process. In the now classic study by Werker and Tees (1984), English-learning infants at 6 to 8 months of age were able to discriminate two syllables from either Hindi or English (see also Werker, Gilbert, Humphrey, \& Tees, 1981). However, unlike the younger infants, older infants maintained discrimination of the English syllables, but failed to discriminate the Hindi syllables. This finding has been replicated with infants from many linguistic environments in terms of various aspects of speech processing. For example, infants learning a tonal language (e.g., Mandarin, Cantonese, and Thai) as their native language show similar narrowing between 6 and 10 months of age (Harrison, 2000; Mattock \& Burnham, 2006; Mattock, Molnar, Polka, \& Burnham, 2008; Yeung, Chen, \& Werker, 2013). Also, infants display narrowing in discriminating vowel contrasts in native versus non-native speech (Kuhl et al., 1992; Moon, Cooper, \& Fifer, 1993; Polka \& Werker, 1994). Consistent with the experiential account, the ability to discriminate non-native speech sounds is maintained at 10 months of age after experimental exposure to non-native speech (Kuhl et al., 2003). Taken together, these studies have established that speech perception in infancy also undergoes narrowing, which is notably similar to that in face perception in terms of both developmental course and timing.

This similarity in narrowing across face and speech perception is consistent with the 
possibility that there are domain-general mechanisms driving early perceptual development across multiple domains (Hadley, Rost, Fava, \& Scott, 2014; Pascalis et al., 2014; Scott et al., 2007). Recent studies suggested several domain-general mechanisms can influence the narrowing, such as individual-level learning (Anzures et al., 2012; Heron-Delaney et al., 2011; Scott \& Monesson, 2009) and selective attention (Markant, Oakes, \& Amso, 2016). By applying these mechanisms to the perception of unfamiliar stimuli (e.g., other-race face and other-species face), post-narrowing infants are able to discriminate these unfamiliar stimuli. However, although these mechanisms do not involve domain specific factor, their efficacies were only examined in face domain. Thus, it is unclear whether the same mechanisms can generally affect the narrowing in other domains, therefore supporting the domain-general hypothesis questionable. In sum, despite the suggestion of domain-general mechanisms that drives the narrowing across domains, the current findings were insufficient to support this hypothesis, mainly because prior studies focused on the narrowing in a single domain.

One way to examine the domain-general hypothesis is to probe the relation between narrowing in two domains. A study for this purpose involve examining narrowing in two domains within each participant (e.g., a within-subject design). A domain-general hypothesis would predict that narrowing in two domains are related. In contrast, if no relation can be found, it might suggest that narrowing is driven by domain-specific mechanisms.

Following this rationale, the current study directly examined the relations between the narrowing of face perception and speech perception with a within-subject design. Within each infant participant, we examined their perceptual discrimination of own-race faces, other-race faces, native speech sounds, and non-native speech sounds. To capture the narrowing in the face 
and speech domains, we recruited infants at 3, 6, 9, and 12 months of age according to previous studies (Maurer \& Werker, 2014).

If narrowing in the two domains is related, there are two possible relations. First, although much of the introduction has made a case for how narrowing in the two domains could be positively related, it is also possible that narrowing in the two domains may be negatively related, possibly reflecting competition between visual faces and auditory speech for domain general processing resources, such as attention. Given that attention is crucial in supporting perceptual development (Amso \& Scerif, 2015; Lewkowicz \& Hansen-Tift, 2012; Markant et al., 2016; Weikum et al., 2007), a competition may occur when the attentional resources are not sufficient to support the development of face and speech perception. The consequence may be a prioritization face over speech information or vice versa. By this view, a negative cross-modal relation would be expected.

This competition account is supported by some recent findings. For example, there is evidence that simultaneous presentation of speech and face information interferes with unimodal perception in young infants (Bahrick, Lickliter, \& Castellanos, 2013). In addition, 8- to 10-month-olds have been found to attend to the mouth region when they look at an articulating face to facilitate speech perception (Hillairet de Boisferon et al., 2017; Lewkowicz \& Hansen-Tift, 2012). In contrast, when no speech information was presented with the face videos or images, infants around the same age allocated their attention to the upper face half (i.e., eye and nose region, Liu et al., 2011; Wheeler et al., 2011; Geangu et al., 2016). Moreover, when encountering a moving face, to what extent infants focus on face versus speech related information varies greatly across infants at 9 months (Xiao, Quinn, Liu, Ge, Pascalis, \& Lee, 
2015). Different infants may allocate their cognitive resources differently to different parts of the face: some may focus on the upper-region of the face to facilitate face perception, whereas others may focus on the lower-region of the face to facilitate speech perception. Infants who prioritize face over speech perception may show earlier (or greater) narrowing in face perception, but delayed (or lesser) narrowing in speech perception and vice versa for those prioritizing speech over face perception. Consequently, such individual differences may contribute to a negative relation between narrowing in the two domains. We refer this negative relation as the Negative Correlation Hypothesis.

Second, there may exist a positive correlation between the narrowing of face and speech perception. This positive relation may reflect a mutual facilitation between the two domains. For example, pairing faces with unique auditory sounds led 9-month-olds to discriminate the faces, which they were unable to discriminate without the uniquely associated auditory signals (Scott \& Monesson, 2009). Similarly, in speech perception, watching an articulating face led to enhanced phonetic discrimination in 6-month-old infants (Teinonen, Aslin, Alku, \& Csibra, 2008). In addition to the notion that development in the face and speech domains may be mutually facilitative, development in both domains may be driven by changes in a third variable (e.g., environmental experience, neural efficiency, attention), also resulting in a positive correlation. We refer to this set of possibilities as the Positive Correlation Hypothesis. It is also possible that if a positive relation were to be observed, then it might be found at a later stage of infancy than the negative relation. This reasoning is based on the idea that attentional resources increase during the infancy period (Reynolds \& Romano, 2016).

Based on the existing evidence of perceptual narrowing in each domain, we expected that 
3-month-olds would discriminate within all four stimulus classes (i.e., own-race faces, other-race faces, native speech, non-native speech). By contrast, infants at or above 6 months of age should only discriminate own-race faces and native speech sounds, but not other-race faces or non-native speech sounds. Furthermore, we used the discrimination advantage of own-race faces relative to that of other-race faces to index perceptual narrowing in face processing. Similarly, we used the discrimination advantage of native speech sounds relative to that of non-native ones to measure narrowing in speech processing. We then performed correlation analyses to probe whether and how differential processing of own- versus other-race faces at each age is related to differential processing of native versus non-native speech. By the Negative Correlation Hypothesis, the expectation would be that narrowing in cross-race face perception should correlate negatively with that in cross-linguistic speech perception. By the Positive Correlation Hypothesis, the expectation would be that narrowing in cross-race face perception should correlate positively with that in cross-linguistic speech perception. However, if no significant relation is found, narrowing in face and speech domains might be driven by domain-specific mechanisms that are independent of one another, where the specific information processed by each domain is not accessible by the other domains (e.g., Fodor, 1983).

\section{Method}

\section{Participants}

Seventy-two Japanese infants participated in the current study, including 18 3-month-olds $\left(M_{\text {age }}=93.22\right.$ days, $S D_{\text {age }}=7.85$ days, 6 females, 12 males $), 186$-month-olds $\left(M_{\text {age }}=182.89\right.$ days, $S D_{\text {age }}=7.99$ days, 8 females, 10 males $), 189$-month-olds $\left(M_{\text {age }}=269.83\right.$ days, $S D_{\text {age }}=$ 
13.73 days, 11 females, 7 males $)$, and 1812 -month-olds $\left(M_{\text {age }}=363.11\right.$ days, $S D_{\text {age }}=8.77$ days, 7 females, 11 males). Sample size was based on our previous studies of perceptual narrowing in infants (e.g., Kelly et al., 2007; Kobayashi et al., 2016; Werker \& Tees, 1984). According to parent reports, these infants had no direct experience with Caucasian individuals or exposure to English speech prior to their participation. Thirty-three infants $\left(n_{3 \text {-months }}=7, n_{6 \text {-months }}=8, n_{9 \text {-months }}\right.$ $=11$, and $n_{12 \text {-months }}=7$ ) also participated in the current study, but were excluded from the data analyses because they failed to complete all of the four conditions. All infants were recruited after their parents expressed willingness to participate in the current study. Although parents did not receive an honorarium for their participation, families were reimbursed for travel expenses. This research was approved by the Ethics Committee of [blinded] (Approval number: 28-P-12).

\section{Materials}

For the face discrimination conditions, two Asian (Japanese) face images were used as own-race face stimuli, and two Caucasian (British) faces were used as other-race face stimuli. All the faces were female adults in frontal view. Moreover, the two faces of each face-race category were similar in their major facial features such as skin color, hair style, and face contour. This ensured that discrimination of faces by infants was based on face identity-relevant cues (e.g., internal facial features), rather than other low-level perceptual cues (e.g., color, shape, and brightness). All of the faces were presented in neutral facial expression. All of the images were resized to the same height of 600 pixels $\left(28.13 \mathrm{~cm}, 26.38^{\circ}\right.$ visual angle at $60 \mathrm{~cm}$ distance). Face image widths varied from 424 to 482 pixels $\left(19.88\right.$ to $22.59 \mathrm{~cm}, 18.81^{\circ}$ to $21.32^{\circ}$ of visual angle at $60 \mathrm{~cm}$ distance), which were set in front of a black background and presented in the center of a 27-inch monitor. 
For the speech sound discrimination tasks, we used two Japanese speech sounds ("kakkou" and "gakkou") as the native speech sounds and two English speech sounds ("dose" and "doze") as the non-native speech sounds. The Japanese speech sounds were recorded from a native female Japanese speaker and the English speech sounds were recorded from a native female English speaker. We chose these native and non-native speech sound pairs for the following reasons: 1) "kakkou" and "gakkou" are common speech components in Japanese and reflect a typical speech sound difference in Japanese, which occurs in the consonant part of the speech sounds; 2) the contrast between the non-native speech sounds is common in English, but never appears in Japanese, and therefore infants would have no experience with this non-native contrast; and 3) our pilot study with adults showed that the contrast in Japanese speech sounds and that in English speech sounds can be reliably detected only by native speakers, but not by non-native speakers.

The intensity of the speech sounds was set to the same level $(65 \mathrm{~dB})$. The sounds were close in duration in both native Japanese (kakkou: $765 \mathrm{~ms}$, and gakkou: $714 \mathrm{~ms}$ ) and non-native English (Dose: 512 ms, Doze: 498 ms). Each sound was played repeatedly. We inserted a silent period between two consecutive occurrences, so as to ensure that the different speech sounds were played at the same pace (once per second). Thus, although each speech sound differed in duration, their frequency of occurrence was the same. These stimulus control measures were used to ensure that infants could not use speech irrelevant perceptual features (e.g., loudness, frequency of occurrence, and sound length) to make the discrimination between speech sounds.

\section{Procedure}

We used a habituation-dishabituation procedure to examine discrimination of faces and 
speech sounds by infants. For the own-race face discrimination condition, infant participants were first habituated with a face image on a series of habituation trials. On each trial, infants saw an own-race Japanese face image at the center of the monitor. Each trial would terminate when infants looked away from the monitor for $2 \mathrm{~s}$ or when they continuously looked at the face for 20 s. Looking time on each trial was calculated by summing up the time the infant looked at the face image. Habituation was reached when average looking time across the last 3 trials was shorter than half of the average looking time on the first three trials. If a participant failed to reach habituation after 20 trials, the program would terminate.

Only if habituation was successfully reached was a dishabituation phase presented. In the dishabituation phase, infants first saw the same habituated face for $5 \mathrm{~s}$, which was then replaced with a novel own-race face for another $5 \mathrm{~s}$. Infant looking times for the habituation and novel faces were recorded in the same way as that for the habituation phase. The procedure for the other-race face condition was identical to that for the own-race face condition except that the habituation and novel faces were other-race Caucasian faces.

For the native speech sound condition, the procedure was exactly the same as that for the face discrimination conditions, except that infants heard the native speech sound from a speaker in front of them and saw a checkerboard on the monitor. Infant looking time to the checkerboard was the index of infant attention to the speech stimuli. In the non-native speech sound condition, infants heard the non-native English speech sounds.

Infant looking behaviors were coded online by an experimenter. During testing, this experimenter sat in a control room, which is next to the testing room, where infants and their parents were. This experimenter could see the infant participants on a computer screen, which 
presented the live images of the infants through a camera placed in front of them. The experimenter was also informed of the start and end of each trial, so as to code the looking behaviors of the infant participants. Given that the test room is soundproof and no experimental content was shown to the experimenter during the test, the experimenter's online coding of infant looking behavior could not be biased. The selection of experimental conditions was controlled by the habituation program, and was thus not known to the experimenter. The computer program automatically calculated and recorded infant looking time according to the experimenter's key pressing. The program was created with E-Prime 2.0.

Every infant participant completed all four conditions: own-race face, other-race face, native speech, and non-native speech. The order of conditions was counterbalanced across participants. The choice of stimuli as habituation or novel was also counterbalanced across participants. A break was given after each condition for at least 2 minutes.

\section{Results}

\section{Habituation Performance}

Table 1 shows the means of the total habituation time and the number of habituation trials for each condition and age group. We first examined whether Stimulus type (Face vs. Speech sound), Experience (Native vs. Non-native), and Age (3, 6, 9, or 12) influenced the total habituation time with a mixed ANOVA. Stimulus type and Experience were within-subject variables, Age was a between-subject continuous variable. Among the examined effects, only the main effect of Age was significant $\left(F[1,70]=8.18, p=.06, \eta^{2}{ }_{\mathrm{P}}=.10\right)$. Post-hoc analysis indicated that 3-month-olds needed longer time to reach habituation than 6-, 9-, and 12-month-olds $(p s<.008)$. No difference in total habituation time was found among 6-, 9-, and 
12-month-olds $(p s>.064)$. No main effect or other interaction was significant $(p s>.105)$.

Moreover, we performed a similar analysis on the number of habituation trials and found no significant main effect or interaction $(p s>.289)$.

Table 1

Mean and SD of Total Habituation Time (s), Number of Habituation Trials, Habituation

Stimulus Looking Time (s) in the Test Phase, and Novel Stimulus Looking Time (s) in the Test

Phase for Each Condition in 3-, 6-, 9-, and 12-month-old Infants

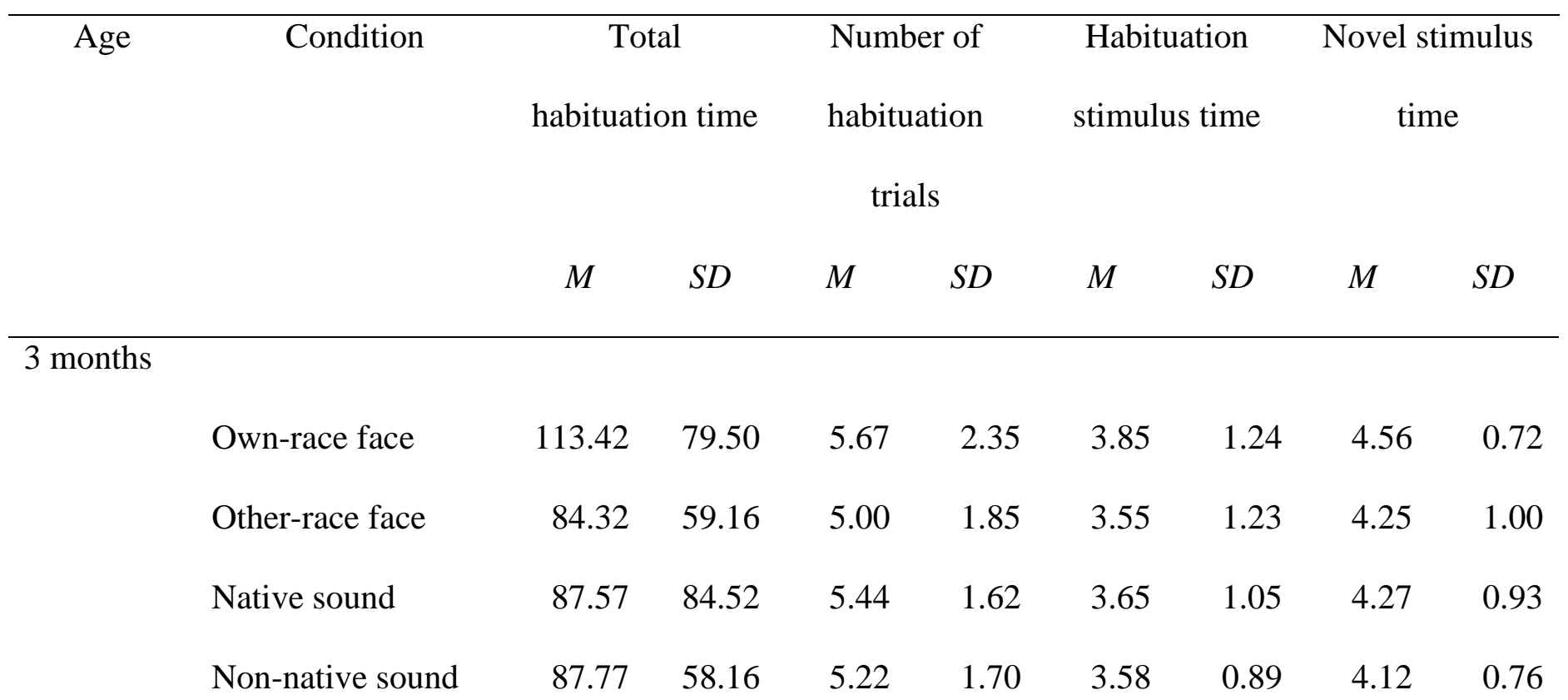

6 months

$\begin{array}{lcccccccc}\text { Own-race face } & 43.70 & 32.88 & 5.56 & 2.04 & 2.78 & 1.04 & 3.60 & 0.97 \\ \text { Other-race face } & 36.66 & 18.58 & 5.44 & 1.69 & 3.33 & 1.12 & 3.50 & 1.05 \\ \text { Native sound } & 42.70 & 25.55 & 5.67 & 2.20 & 3.11 & 0.95 & 3.82 & 0.91 \\ \text { Non-native sound } & 46.20 & 24.67 & 5.61 & 2.25 & 3.59 & 1.13 & 3.77 & 1.02\end{array}$


9 months

$\begin{array}{lcccccccc}\text { Own-race face } & 54.57 & 30.29 & 5.17 & 1.86 & 3.35 & 1.31 & 4.14 & 1.05 \\ \text { Other-race face } & 46.20 & 32.90 & 4.28 & 0.67 & 3.44 & 0.86 & 3.79 & 1.03 \\ \text { Native sound } & 40.98 & 18.49 & 4.83 & 1.25 & 3.07 & 0.95 & 3.65 & 1.03 \\ \text { Non-native sound } & 45.82 & 23.43 & 5.50 & 1.69 & 3.61 & 1.20 & 3.23 & 1.15\end{array}$

12 months

$\begin{array}{lcccccccc}\text { Own-race face } & 55.84 & 26.09 & 5.33 & 1.68 & 3.36 & 1.27 & 4.43 & 0.92 \\ \text { Other-race face } & 58.16 & 40.82 & 4.94 & 1.39 & 3.80 & 1.18 & 4.18 & 0.87 \\ \text { Native sound } & 71.74 & 47.84 & 5.89 & 1.75 & 4.05 & 0.84 & 4.56 & 0.60 \\ \text { Non-native sound } & 51.97 & 47.12 & 4.61 & 0.78 & 3.78 & 1.03 & 3.50 & 1.30\end{array}$

\section{Dishabituation Performance}

We first calculated the dishabituation score to represent infant discrimination performance for own- and other-race faces and native and non-native sounds. The dishabituation score is calculated by subtracting the looking time for the habituated stimulus from that for the novel stimulus on the dishabituation trials (see Table 1). A dishabituation score higher than 0 indicates that infants were able to discriminate the novel stimulus from the habituation stimulus.

A Stimulus type (Face vs. Speech sound), Experience (Native vs. Non-native), Age (3, 6, 9, or 12), and Gender (Female vs. Male) mixed ANOVA indicated that infant gender did not significantly affect dishabituation performance for faces or sounds ( $p$ s > .528). We therefore collapsed the data across gender for the following analyses.

We also examined whether order of the 4 conditions affected dishabituation performance 
with a Stimulus type (Face vs. Speech sound), Experience (Native vs. Non-native), Age (3, 6, 9, or 12), and Order (1st, 2nd, 3rd, or 4th) ANOVA. It should be noted that one condition could be carried out only at one of the 4 order places, which led the Stimulus type and Experience to become between-subject variables. The results showed no main effect of Order or interactions including Order reached significance $(p s>.071)$, suggesting that the order of the 4 conditions did not affect discrimination performance of the infants.

We next examined whether Stimulus type (Face vs. Speech sound), Experience (Native vs. Non-native), and Age (3, 6, 9, or 12) influenced the dishabituation score with a mixed ANOVA. Stimulus type and Experience were within-subject variables, and Age was a between-subject continuous variable. Only the interaction between Age and Experience was significant $\left(F[1,70]=4.38, p=.040, \eta^{2}=.06\right)$, suggesting the difference between discriminating own-race/native and other-race/non-native stimuli was affected by the age of the participants. In particular, the significant interaction indicates the emergence of narrowing in the perception of faces and sounds. Moreover, we found no other main effect or interaction significantly affected the dishabituation score $(p s>.121)$. These outcomes suggest that perceptual narrowing in face and speech sound perception did not differ across the two domains.

To further probe the emergence of narrowing, we examined whether infants of different ages were able to discriminate the new and habituated stimulus for each stimulus type by performing a series of one-sample $t$-tests against zero for each condition at each age. As shown in Figure 1, the dishabituation score for the 3-month-olds was significantly above 0 in the own-race face condition $(M=0.71 \mathrm{~s}, S E=0.24 \mathrm{~s}, t[17]=3.2, p=.005)$, the other-race face condition $(M=0.70 \mathrm{~s}, S E=0.29 \mathrm{~s}, t[17]=2.64, p=.017)$, the native-sound condition $(M=0.62$ 
$\mathrm{s}, S E=0.17 \mathrm{~s}, t[17]=3.87, p=.001)$, and the non-native sound condition $(M=0.54 \mathrm{~s}, S E=0.22$ $\mathrm{s}, t[17]=2.59, p=.019)$. These results indicate that infants at 3 months of age were able to discriminate among faces from their own-race group as well as among faces from the other-race group. They were also able to discriminate among native speech sounds and among non-native speech sounds.

The dishabituation scores for the 6-month-olds were significantly above 0 in the own-race face condition $(M=0.82 \mathrm{~s}, S E=0.27 \mathrm{~s}, t[17]=3.28, p=.004)$ and the native-sound condition $(M=0.71 \mathrm{~s}, S E=0.25 \mathrm{~s}, t[17]=3.03, p=.008)$. The one-sample $t$-tests for the 6-month-olds, however, failed to reached significance in both the other-race face condition ( $M=$ $0.16 \mathrm{~s}, S E=0.25 \mathrm{~s}, t[17]=0.72, p=.480)$ and the non-native sound condition $(M=0.18 \mathrm{~s}, S E=$ $0.17 \mathrm{~s}, t[17]=1.18, p=.254)$. Thus, 6 -month-olds could discriminate own-race faces and native speech sounds but not other-race faces and non-native sounds, suggesting that narrowing had occurred by this age.

Similar to 6-month-olds, 9- and 12-month-olds only showed dishabituation scores that were significantly above 0 in the own-race face condition (9-months: $M=0.79 \mathrm{~s}, S E=0.26 \mathrm{~s}$, $t[17]=3.34, p=.004 ; 12$-months: $M=1.08 \mathrm{~s}, S E=0.39 \mathrm{~s}, t[17]=2.97, p=.009)$ and native sound condition (9-months: $M=0.57 \mathrm{~s}, S E=0.22 \mathrm{~s}, t[17]=2.76, p=.013 ; 12$-months: $M=0.52$ $\mathrm{s}, S E=0.21 \mathrm{~s}, t[17]=2.68, p=.016)$, but not in the other-race face condition (9-months: $M=$ $0.35 \mathrm{~s}, S E=0.34 \mathrm{~s}, t[17]=1.13, p=.276 ; 12$-months: $M=0.38 \mathrm{~s}, S E=0.26 \mathrm{~s}, t[17]=1.6, p$ $=.128$ ) or non-native sound condition (9-months: $M=-0.37 \mathrm{~s}, S E=0.24 \mathrm{~s}, t[17]=-1.64, p$ $=.120 ; 12$-months: $M=-0.28 \mathrm{~s}, S E=0.29 \mathrm{~s}, t[17]=-1.05, p=.306)$. These results indicate that the narrowing that was observed beginning at 6 months was also in evidence at 9 and 12 months 
of age.
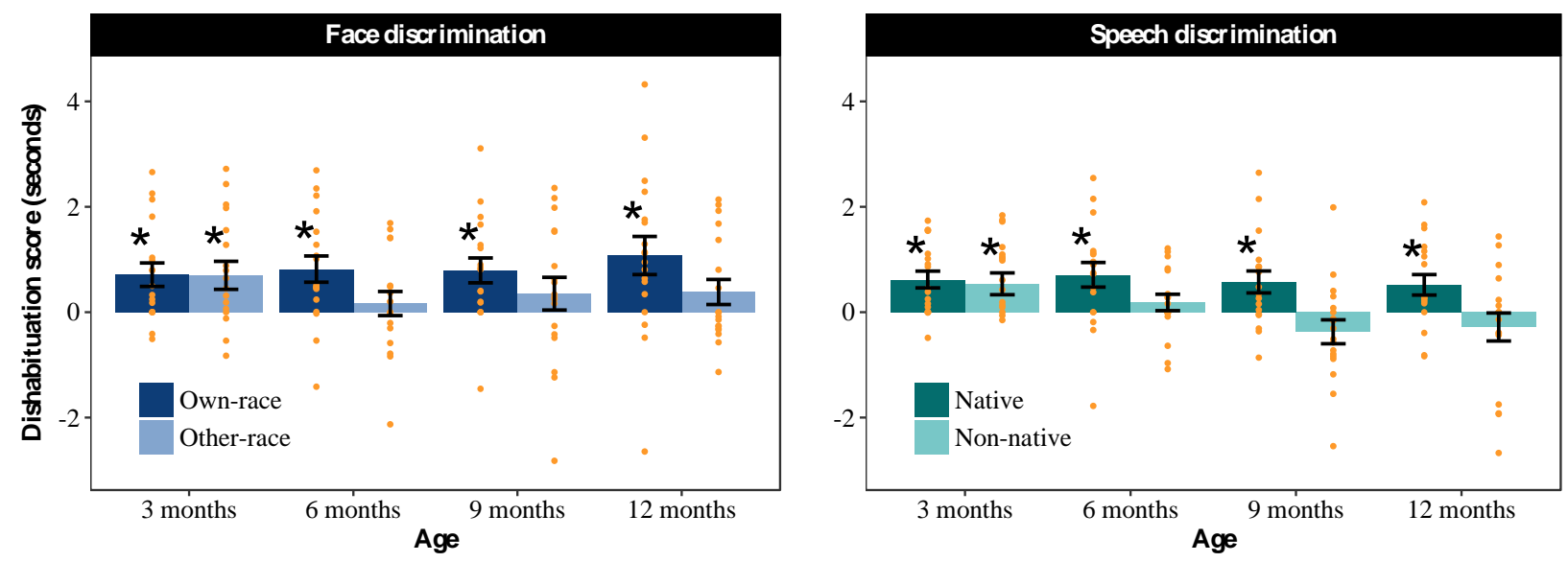

Figure 1. Mean dishabituation scores (seconds) for each condition in 3-, 6-, 9-, and 12-month-old infants. The length of each error bars represents one standard error of the mean. The orange dots represent each participant's dishabituation score for each condition. Asterisks indicate that the dishabituation score is significantly different from $0(p<.05)$.

Together, the results indicate that infants at 3 months of age exhibited a broad sensitivity to the contrasts in faces from own- and other-race categories. They were also sensitive to the phonetic contrasts in native and non-native speech. Six-, 9-, and 12-month-old infants, however, could only discriminate faces from their own-race category and native speech sounds, but not the other-race faces or non-native sounds. The findings provide evidence of perceptual narrowing in the development of visual and auditory perception in the second half of the first year of life, with emergence of narrowing at 6 months.

A possible critique of the native Japanese sounds used in our investigation is that "gakkou" means school and "kakkou" means look/appearance. It could be the case that older infants are more familiar with "gakkou", therefore helping them discriminate it from "kakkou", which infants are less likely to hear. In other words, older infants may have discriminated the 
two native Japanese sounds due to difference in familiarity with each word, rather than their specialized auditory perception. However, if there had existed a familiarity difference between the two native sounds, we would have expected a difference in habituation rate or habituation time between the participants who were habituated with "gakkou" and tested with "kakkou" and the participants who were habituated with "kakkou" and tested with "gakkou". More specifically, the habituation rate or time should be faster in the "gakkou" habituation participants than the "kakkou" habituation participants, given that "gakkou" (school) could be more familiar to infants than "kakkou" (look/appearance). To this end, we used independent two-sample $t$-tests to compare the time it took the "gakkou" habituation and "kakkou" habituation participants to reach the habituation criterion. The results showed no significant difference between the two habituation sounds in any age group (3-month-olds: $M_{\text {gakkou }}=78.17, S D_{\text {gakkou }}=62.08, M_{\text {kakkou }}=$ 94.59, $S D_{\text {kakkou }}=94.86, t[16]=0.36, p=.727 ; 6$-month-olds: $M_{\text {gakkou }}=29.10, S D_{\text {gakkou }}=15.42$, $M_{\text {kakkou }}=51.35, S D_{\text {kakkou }}=27.48, t[16]=1.94, p=.070 ; 9$-month-olds: $M_{\text {gakkou }}=48.18, S D_{\text {gakkou }}$ $=14.49, M_{\text {kakkou }}=38.56, S D_{\text {kakkou }}=20.73, t[16]=0.94, p=.359 ; 12$-month-olds: $M_{\text {gakkou }}=44.88$, $\left.S D_{\text {gakkou }}=22.16, M_{\text {kakkou }}=77.99, S D_{\text {kakkou }}=54.86, t[16]=1.29, p=.216\right)$. These outcomes do not provide evidence that the infant participants in the current study were responding to the familiarity difference between the two native sounds. The discrimination of the two sounds is thus more likely attributable to the perceptual differences between the sounds.

\section{Relations Between Face and Speech Processing}

We next examined the relation between perceptual narrowing in discriminating faces and speech sounds. We calculated perceptual narrowing scores in face discrimination by subtracting the dishabituation score for the other-race face condition from that for the own-race face 
condition in each participant $\left(\right.$ Narrowing $_{\text {face }}=$ Dishabituation $_{\text {own-race }}-$ Dishabituation $\left._{\text {other-race }}\right)$. We calculated the narrowing score in infant speech perception with a similar formula $\left(\right.$ Narrowing $_{\text {speech }}=$ Dishabituation $_{\text {native }}-$ Dishabituation $\left._{\text {non-native }}\right)$.

We examined the relation between narrowing in face and speech perception with a multi-variable linear regression analysis. Because this analysis focused on the relation between narrowing in the two modalities, it was conducted on the age groups in which narrowing was observed. To this end, this multi-variable linear regression included data from 6-, 9-, and 12-month-olds. Specifically, we designated narrowing in speech processing for each participant as the output variable, and narrowing in face processing, participant age in months, and their interaction as predictive variables. If the relation changes with age, we should find a significant interaction.

As shown in Table 2, the regression revealed a significant effect of narrowing in face processing $(t=-2.88, p=.006)$, indicating that narrowing in face and speech processing was correlated among infants from 6 to 12 months of age. Moreover, we found a significant interaction between age group and narrowing in face processing $(t=3.05, p=.004)$, suggesting that the relation differed among 6-, 9-, and 12-month-olds.

To further probe the interaction, we calculated the Pearson linear correlations for each age group. The results showed that narrowing in face and speech processing did not correlate at 6 months of age (Figure 2, $r=-.18, p=.483)$. The two were negatively correlated at 9 months $(r=$ $-.63, p=.005)$, and at 12 months, the two were positively correlated $(r=.62, p=.006)$. These results thus reveal age-related changes in the relation between narrowing in face and speech processing from 6 to 12 months of age. 
Although 3-month-olds did not show narrowing, we still computed their narrowing scores, and performed a similar correlational analysis as above. We found that, as expected and like the 6-month-olds, the narrowing scores in face and speech processing did not correlate with each other at 3 months of age $(r=.27, p=.270)$.
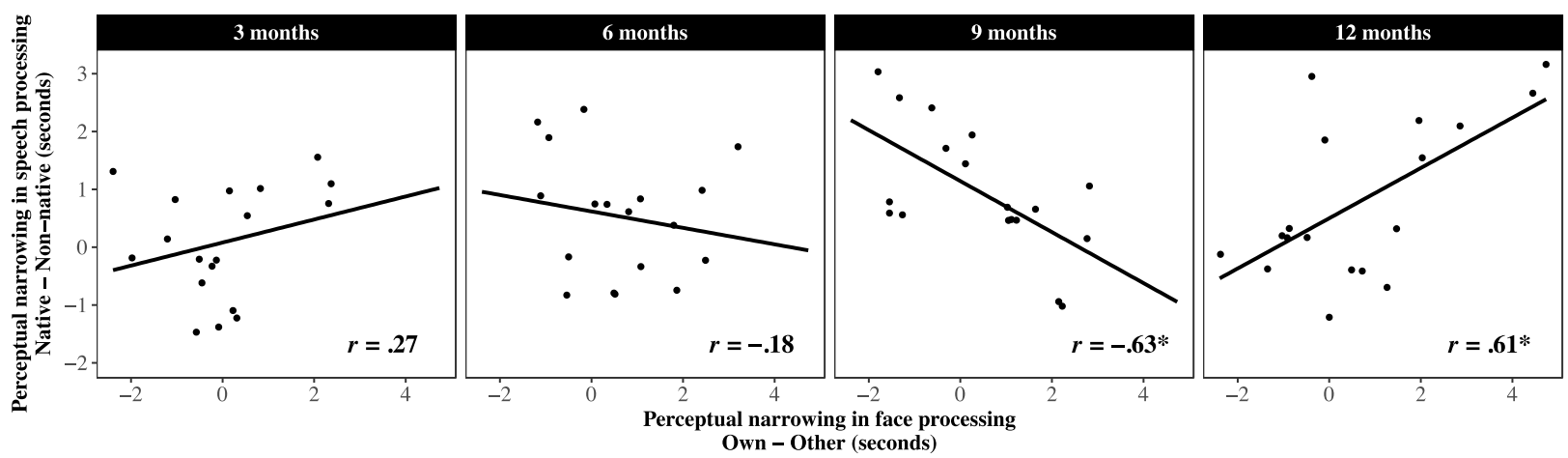

Figure 2. The relation between narrowing in face and speech perception in 3-, 6-, 9-, and 12-month-old infants. Each dot represents a participant. Each line represents the linear relation between narrowing in face and speech processing. The values at the bottom right corner indicate Pearson correlation coefficients for the relations. Asterisks indicate that the relation is significant $(p<.05)$

Table 2. Summary of the multi-variable linear regression predicting the speech expertise scores. Asterisk indicates a significant effect $(p<.050)$

\begin{tabular}{lccc}
\hline Variables & $B$ & SE B & $p$ \\
\hline Face expertise score & -1.57 & 0.40 & $.006^{*}$ \\
Age & -0.04 & 0.07 & .595 \\
Interaction & 0.12 & 0.04 & $.004^{*}$ \\
\hline
\end{tabular}




\begin{tabular}{ll}
\hline$R^{2}$ & .17 \\
$F$ for change in $R^{2}$ & 3.35 \\
\hline
\end{tabular}

\section{Discussion}

The current study directly examined the relation between perceptual narrowing in the face and speech domains in infants at 3, 6, 9, and 12 months of age with a within-subject design. We replicated the narrowing with the current experiment design, where infants exhibited narrowing in the both domains around 6 months of age. Moreover, by concurrently testing infants in the discrimination of own- and other-race faces and native and non-native speech, we found that narrowing in the domains of face and speech perception were not correlated at 6 months of age, negatively correlated at 9 months, and positively correlated at 12 months.

The result that Japanese infants discriminated own- and other-race faces differently at different ages is consistent with prior findings on the development of face perception in infancy (Maurer \& Werker, 2014). In addition to being observed for own- versus other-race faces, narrowing in face perception has also been reported in studies of own- versus other-species faces (Pascalis et al., 2002; Pascalis et al., 2005; Scott \& Monesson, 2009), and adult versus infant faces (Kobayashi et al., 2016; Macchi Cassia et al., 2013). Thus, the developmental pattern found in the present and previous studies together demonstrates that narrowing is a perceptual phenomenon that can be observed across multiple face attributes for which there are differences in the frequency of experience.

We also found perceptual narrowing in speech perception by Japanese infants. This finding is consistent with studies that have reported narrowing in perceptual development for 
multiple linguistic groups and various speech components, such as vowels (Kuhl et al., 1992; Moon et al., 1993; Polka \& Werker, 1994), consonants (for a review, see Werker \& Curtin, 2005), and lexical tones (Harrison, 2000; Mattock \& Burnham, 2006; Mattock et al., 2008; Yeung et al., 2013). Together, the findings suggest that the auditory system of infants gradually becomes specialized to respond to familiar speech and tune out unfamiliar speech.

The observed narrowing in face and speech domains is consistent with development in other domains, such as the perception of musical rhythm (Hannon \& Trehub, 2005; Lynch, Eilers, Oller, \& Urbano, 1990), and inter-sensory correspondence (Lewkowicz \& Ghazanfar, 2006; Pons et al., 2009). The similarity among domains in the first year of life suggests that narrowing is a general phenomenon in infancy.

The within-subject design allowed us to directly examine whether perceptual narrowing in one domain is related to that in another. By performing correlational analyses at each age, we observed age-related changes in this relation. Specifically, at 3 months of age, when perceptual narrowing was not yet in evidence in either domain, the extent of narrowing in one domain was not surprisingly unrelated to that in the other. At 6 months, when narrowing in both domains had begun, a significant correlation between the extent of narrowing in the two domains was still not present, suggesting that narrowing in the face and speech domains are not yet linked at their outset.

At 9 months of age, we found a significant negative correlation between the extent of perceptual narrowing in the two domains, which is in accord with the Negative Correlation Hypothesis. Infants who showed greater narrowing in face perception displayed lesser narrowing in speech perception, and vice versa. One plausible interpretation is that a competition exists 
between the development of face and speech processing systems as they vie for attentional capacity as a limited resource. This competition account is in accord with the nature of face and speech experiences, which are highly inter-connected. A competition may occur when infants are not capable of simultaneously processing face and speech information equally well.

This competition of attentional resources is revealed in the visual attention pattern infants display for articulating faces. Infants at 8 to 10 months of age use visual information to assist speech perception by allocating attention to the mouth region (e.g., Hillairet de Boisferon et al., 2016; Lewkowicz \& Hansen-Tift, 2012). A recent imaging study found that the mouth looking tendency positively correlated with the brain activity on the left temporal region, which represents speech perception (Altvater-Mackensen \& Grossmann, 2016). Infants at this age also attended to the eye and nose regions to support face processing (e.g., Liu et al., 2011; Liu et al., in press; Wheeler et al., 2011; Geangu et al., 2016). Given limited attentional resources in infants at this age range (e.g., Reynolds \& Romano, 2016), different attention patterns may indicate a competition of attention for processing face or speech related information. Especially, different infants may allocate their attention differently to different parts of the faces (Xiao et al., 2015). Such individual differences may lead some infants to develop narrowing in the face domain prior to that in the speech domain, and vice versa for other infants.

In contrast to the results with 9-month-olds, we found a positive relation between narrowing of own- and other-race face perception and that of native and non-native speech perception at 12 months of age. This finding supports the Positive Correlation Hypothesis. The developmental change between the negative relation at 9 months and the positive relation at 12 months is consistent with the idea that face perception may no longer compete with speech 
perception for cognitive resources in the older age group. In accord with this hypothesis, it has been reported that 12-month-olds can process speech (Lewkowicz \& Hansen-Tift, 2012) while also attending to the eye region, with the latter presumably facilitating face processing. This facilitation may be achieved by an intermodal top-down process (Emberson, Richards, \& Aslin, 2015; Hadley et al., 2014). For example, Emberson and colleagues (2015) have reported that experience with associated audio and visual events establishes a kind of Hebbian cortical association across domains, in which stimulation in one domain leads to cortical activation in another. Given that face and speech are highly inter-connected in real-life contexts, infants may establish cortical associations between the two types of stimuli. Moreover, this cross-domain connection at the cortical level was found in infants at 6 months of age, which is earlier than the age of the positive correlation in the current study (i.e., 12 months). This timing difference suggests that the cross-modal cortical association may support the later developed coordination and integration in perception between modalities. In this way, development in one domain would activate and facilitate development in the other domain. In addition to the possibility of mutual facilitation between domains, it could also be the case that both the face and speech domains benefit from development of one or more "third" variables (e.g., development of attentional capacity, cumulative experience, or neural efficiency).

Why did perceptual narrowing for each modality emerge at 6 months with the emergence of a relation between modalities occurring only from 9 months onward? One possibility is that the perceptual development in each domain may trigger intermodal competition at 9 months of age, and coordination and integration between modalities at 12 months of age. However, because the current study only used a cross-sectional design, we are unable to address this possibility. 
Future studies could use a longitudinal design to further examine the mechanisms driving the developmental relations among domains. Specifically, one could examine the cross-domain relations not only within a particular age, but also across different ages. A longitudinal design would allow one to examine, for example, whether face processing at a younger age is related to speech processing at an older age, or vice versa. This type of cross-age relational analysis would offer a deeper understanding of the dynamics of perceptual development across domains. One interesting possibility that awaits empirical confirmation is if processing resources that are deployed to facilitate development in one domain at a given age are then reallocated to another domain at an older age.

It should be noted that the present study focused on the relation between narrowing of face and speech, and that these two domains are naturally connected. Thus, it is unclear whether the developmental relations between the two domains reflect this specific interconnection or represent a more general developmental phenomenon. To this extent, the data with the 9- and 12-month-olds are consistent accounts of development positing domain-general mechanisms or domain-specific mechanisms that interact. In order to address this specific question, future studies are needed to investigate cross-modal relations between domains that are not as inherently linked as face and speech (e.g., face and music). Given that narrowing has been evidenced in the domain of music processing (Hannon \& Trehub, 2005; Lynch et al., 1990), observing similar relations between narrowing in the face and music modalities would suggest a general phenomenon in the early perceptual development of cross-modal relations.

Another related question that needs to be addressed in future studies is whether the developmental pattern of relations between domains seen here is limited to infancy or is a 
broader phenomenon that can be observed at later periods of development. As noted in the introduction, several studies with children from early and middle childhood have reported relations between face and print word processing (Dundas, Plaut, \& Behrmann, 2013, 2014a, 2014b; Li et al., 2013). For example, Li and colleagues (2013) found that print and face processing were negatively related in young children who had just begun to read and were still developing the ability to process faces. However, after entering elementary school, with further development of face and print processing, children's face and print processing were positively correlated (Dundas et al., 2013). Both the current infant and prior child data suggest that intermodal relations may develop from unrelated, to competitive, to cooperative, thereby implying that this pattern may be observed at multiple periods of development. This developmental pattern echoes the notion of "vertical décalage", in which a cognitive operation occurring at an early stage of development is observed again at a later point in development (Piaget, 1950).

One limitation in the experimental design that should be acknowledged is that only one pair of stimuli was used to examine performance in each of the four cells (i.e., own race, other race, native speech, non-native speech). We specifically used a single stimulus pair design to ensure that the correlational results in the four age groups would not be harmed by variation across different stimulus exemplars. Future studies can examine the generalizability of the relations we observed by using different stimulus contrasts.

To conclude, the current study investigated perceptual narrowing in face and speech processing in 3-, 6-, 9-, and 12-month-old Japanese infants. The results indicated that infants began to show narrowing in face and speech discrimination at 6 months of age. Moreover, by 
concurrently testing the ability of infants to discriminate own- and other-race faces and native and non-native speech, we found that narrowing in face and speech perception were not correlated at 6 months of age, negatively correlated at 9 months, and positively correlated at 12 months. The evidence suggests a developmental pattern of intermodal relations, which develops from unrelated, to negative, to positive. The findings imply that perception in different domains becomes interdependent during the course of infancy, and that the nature of that interdependence changes as development unfolds. 


\section{References}

Altvater-Mackensen, N., \& Grossmann, T. (2016). The role of left inferior frontal cortex during audiovisual speech perception in infants. Neuroimage, 133, 14-20.

Amso, D., \& Scerif, G. (2015). The attentive brain: Insights from developmental cognitive neuroscience. Nature Reviews: Neuroscience, 16, 606-619.

Anzures, G., Wheeler, A., Quinn, P. C., Pascalis, O., Slater, A. M., Heron-Delaney, M., et al. (2012). Brief daily exposures to Asian females reverses perceptual narrowing for Asian faces in Caucasian infants. Journal of Experimental Child Psychology, 112, 484-495.

Bahrick, L. E., Lickliter, R., \& Castellanos, I. (2013). The development of face perception in infancy: Intersensory interference and unimodal visual facilitation. Developmental Psychology, 49, 1919-1930.

Behrmann, M., \& Plaut, D. C. (2013). Distributed circuits, not circumscribed centers, mediate visual recognition. Trends in Cognitive Sciences, 17, 210-219.

Cashon, C. H., Ha, O.-R., Allen, C. L., \& Barna, A. C. (2013). A U-shaped relation between sitting ability and upright face processing in infants. Child Development, 84, 802-809.

Chien, S. H., Wang, J. F., \& Huang, T. R. (2016). Developing the own-race advantage in 4-, 6-, and 9-month-old taiwanese infants: A perceptual learning perspective. Frontiers in Psychology, 7, 1606.

Dundas, E. M., Plaut, D. C., \& Behrmann, M. (2013). The joint development of hemispheric lateralization for words and faces. Journal of Experimental Psychology: General, 142, 348-358.

Dundas, E. M., Plaut, D. C., \& Behrmann, M. (2014a). An ERP investigation of the 
co-development of hemispheric lateralization of face and word recognition. Neuropsychologia, 61, 315-323.

Dundas, E. M., Plaut, D. C., \& Behrmann, M. (2014b). Variable left-hemisphere language and orthographic lateralization reduces right-hemisphere face lateralization. Journal of Cognitive Neuroscience, 27, 1-13.

Emberson, L. L., Richards, J. E., \& Aslin, R. N. (2015). Top-down modulation in the infant brain: Learning-induced expectations rapidly affect the sensory cortex at 6 months. Proceedings of the National Academy of Sciences of the United States of America, 112, 201510343-201519590.

Flom, R. (2014). Perceptual narrowing: Retrospect and prospect. Developmental Psychobiology, $56,1442-1453$.

Fodor, J. A. (1983). The modularity of mind. Cambridge, MA: MIT Press.

Geangu, E., Ichikawa, H., Lao, J., Kanazawa, S., Yamaguchi, M. K., Caldara, R., \& Turati, C. Culture shapes 7-month-olds' perceptual strategies in discriminating facial expressions of emotion. Current Biology, 26, R663-R664.

Hadley, H., Rost, G. C., Fava, E., \& Scott, L. S. (2014). A mechanistic approach to cross-domain perceptual narrowing in the first year of life. Brain Sciences, 4, 613-634.

Hannon, E. E., \& Trehub, S. E. (2005). Tuning in to musical rhythms: Infants learn more readily than adults. Proceedings of the National Academy of Sciences, 102, 12639-12643.

Harrison, P. (2000). Acquiring the phonology of lexical tone in infancy. Lingua, 110, 581-616. Heron-Delaney, M., Anzures, G., Herbert, J. S., Quinn, P. C., Slater, A. M., Tanaka, J. W., et al. (2011). Perceptual training prevents the emergence of the other race effect during infancy. 
PLOS ONE, 6, e19858.

Hillairet de Boisferon, A., Tift, A. H., Minar, N. J., \& Lewkowicz, D. J. (2017). Selective attention to a talker's mouth in infancy: Role of audiovisual temporal synchrony and linguistic experience. Developmental Science, 20, doi: 10.1111/desc.12381

Kelly, D. J., Liu, S., Lee, K., Quinn, P. C., Pascalis, O., Slater, A. M., \& Ge, L. (2009). Development of the other-race effect during infancy: Evidence toward universality? Journal of Experimental Child Psychology, 104, 105-114.

Kelly, D. J., Quinn, P. C., Slater, A. M., Lee, K., Ge, L., \& Pascalis, O. (2007). The other-race effect develops during infancy: Evidence of perceptual narrowing. Psychological Science, 18, 1084-1089.

Kobayashi, M., Macchi Cassia, V., Kanazawa, S., Yamaguchi, M. K., \& Kakigi, R. (2016). Perceptual narrowing towards adult faces is a cross-cultural phenomenon in infancy: A behavioral and near-infrared spectroscopy study with Japanese infants. Developmental Science, 21, e12498. doi:10.1111/desc. 12498

Kuhl, P. K., Tsao, F.-M., \& Liu, H.-M. (2003). Foreign-language experience in infancy: Effects of short-term exposure and social interaction on phonetic learning. Proceedings of the National Academy of Sciences of the United States of America, 100, 9096-9101.

Kuhl, P., Williams, K., Lacerda, F., Stevens, K., \& Lindblom, B. (1992). Linguistic experience alters phonetic perception in infants by 6 months of age. Science, 255, 606-608.

Le Grand, R., Mondloch, C. J., Maurer, D., \& Brent, H. P. (2001). Early visual experience and face processing. Nature, 410, 890-890.

Lewkowicz, D. J., \& Ghazanfar, A. A. (2006). The decline of cross-species intersensory 
perception in human infants. Proceedings of the National Academy of Sciences, 103, $6771-6774$.

Lewkowicz, D. J., \& Ghazanfar, A. A. (2009). The emergence of multisensory systems through perceptual narrowing. Trends in Cognitive Sciences, 13, 470-478.

Lewkowicz, D. J., \& Hansen-Tift, A. M. (2012). Infants deploy selective attention to the mouth of a talking face when learning speech. Proceedings of the National Academy of Sciences, 109, 1431-1436.

Li, S., Lee, K., Zhao, J., Yang, Z., He, S., \& Weng, X. (2013). Neural competition as a developmental process: Early hemispheric specialization for word processing delays specialization for face processing. Neuropsychologia, 51, 950-959.

Liu, S, Quinn, P. C., Xiao, N. G., Wu, Z., Liu, G., \& Lee, K. (in press). Relations between scanning and recognition of own- and other-race faces in 6- and 9-month-old infants. PsyCh Journal.

Liu, S., Quinn, P. C., Wheeler, A., Xiao, N. G., Ge, L., \& Lee, K. (2011). Similarity and difference in the processing of same- and other-race faces as revealed by eye tracking in 4- to 9-month-olds. Journal of Experimental Child Psychology, 108, 180-189.

Liu, S., Xiao, N. G., Quinn, P. C., Zhu, D., Ge, L., Pascalis, O., \& Lee, K. (2015). Asian infants show preference for own-race but not other-race female faces: The role of infant caregiving arrangements. Frontiers in Psychology, 6, 593.

Lynch, M. P., Eilers, R. E., Oller, D. K., \& Urbano, R. C. (1990). Innateness, experience, and music perception. Psychological Science, 1, 272-276.

Macchi Cassia, V., Bulf, H., Quadrelli, E., \& Proietti, V. (2013). Age-related face processing 
bias in infancy: Evidence of perceptual narrowing for adult faces. Developmental Psychobiology, 56, 238-248.

Markant, J., Oakes, L. M., \& Amso, D. (2016). Visual selective attention biases contribute to the other-race effect among 9-month-old infants. Developmental Psychobiology, 58, $355-365$.

Mattock, K., \& Burnham, D. (2006). Chinese and English infants' tone perception: Evidence for perceptual reorganization. Infancy, 10, 241-265.

Mattock, K., Molnar, M., Polka, L., \& Burnham, D. (2008). The developmental course of lexical tone perception in the first year of life. Cognition, 106, 1367-1381.

Maurer, D., \& Werker, J. F. (2014). Perceptual narrowing during infancy: A comparison of language and faces. Developmental Psychobiology, 56, 154-178.

Moon, C., Cooper, R. P., \& Fifer, W. P. (1993). Two-day-olds prefer their native language. Infant Behavior and Development, 16, 495-500.

Pascalis, O., de Haan, M., \& Nelson, C. A. (2002). Is face processing species-specific during the first year of life? Science, 296, 1321-1323.

Pascalis, O., Løvenbruck, H., Quinn, P. C., Kandel, S., Tanaka, J. W., \& Lee, K. (2014). On the links among face processing, language processing, and narrowing during development. Child Development Perspectives, 8, 65-70.

Pascalis, O., Scott, L. S., Kelly, D. J., Shannon, R. W., Nicholson, E., Coleman, M., \& Nelson, C. A. (2005). Plasticity of face processing in infancy. Proceedings of the National Academy of Sciences, 102, 5297-5300.

Piaget, J. (1950). The psychology of intelligence. London: Routledge \& Kegan Paul. 
Polka, L., \& Werker, J. F. (1994). Developmental changes in perception of nonnative vowel contrasts. Journal of Experimental Psychology: Human Perception and Performance, 20, $421-435$.

Pons, F., Lewkowicz, D. J., Soto-Faraco, S., \& Sebastián-Gallés, N. (2009). Narrowing of intersensory speech perception in infancy. Proceedings of the National Academy of Sciences of the United States of America, 106, 10598-10602.

Quinn, P. C., Lee, K., Pascalis, O., \& Tanaka, J. W. (2016). Narrowing in categorical responding to other-race face classes by infants. Developmental Science, 19, 362-371.

Rennels, J. L., \& Davis, R. E. (2008). Facial experience during the first year. Infant Behavior and Development, 31, 665-678.

Reynolds, G. D., \& Romano, A. C. (2016) The development of attention systems and working memory in infancy. Frontiers in Systems Neuroscience, 10:15. doi: 10.3389/fnsys.2016.00015

Scott, L. S., \& Monesson, A. (2009). The origin of biases in face perception. Psychological Science, 20, 676-680.

Scott, L. S., Pascalis, O., \& Nelson, C. A. (2007). A domain-general theory of the development of perceptual discrimination. Current Directions in Psychological Science, 16, 197-201.

Sugden, N. A., \& Marquis, A. R. (2017). Meta-analytic review of the development of face discrimination in infancy: Face race, face gender, infant age, and methodology moderate face discrimination. Psychological Bulletin, 143, 1201-1244.

Sugden, N. A., Mohamed-Ali, M. I., \& Moulson, M. C. (2014). I spy with my little eye: Typical, daily exposure to faces documented from a first-person infant perspective. 
Developmental Psychobiology, 56, 249-261.

Teinonen, T., Aslin, R. N., Alku, P., \& Csibra, G. (2008). Visual speech contributes to phonetic learning in 6-month-old infants. Cognition, 108, 850-855.

Tham, D. S. Y., Bremner, J. G., \& Hay, D. (2015). In infancy the timing of emergence of the other-race effect is dependent on face gender. Infant Behavior and Development, 40, $131-138$.

Weikum, W. M., Vouloumanos, A., Navarra, J., Soto-Faraco, S., Sebastián-Gallés, N., \& Werker, J. F. (2007). Visual language discrimination in infancy. Science, 316, 1159-1159.

Werker, J. F., \& Tees, R. C. (1984). Cross-language speech perception: Evidence for perceptual reorganization during the first year of life. Infant Behavior and Development, 7, 49-63.

Werker, J. F., Gilbert, J. H. V., Humphrey, K., \& Tees, R. C. (1981). Developmental aspects of cross-language speech perception. Child Development, 52, 349.

Wheeler, A., Anzures, G., Quinn, P. C., Pascalis, O., Omrin, D. S., \& Lee, K. (2011). Caucasian infants scan own- and other-race faces differently. PLoS ONE, 6, e18621.

Xiao, N. G., Quinn, P. C., Liu, S., Ge, L., Pascalis, O., \& Lee, K. (2015). Eye tracking reveals a crucial role for facial motion in recognition of faces by infants. Developmental Psychology, 51, 744-757.

Yeung, H. H., Chen, K. H., \& Werker, J. F. (2013). When does native language input affect phonetic perception? The precocious case of lexical tone. Journal of Memory and Language, 68, 123-139. 